\title{
Cancer stem cells in liver metastasis from colon adenocarcinoma express components of the renin- angiotensin system
}

\author{
Anantha Narayanan ${ }^{1,2}$, Susrutha K. Wickremesekera ${ }^{1,2}$, Bede van Schaijik ${ }^{1}$, Reginald W. Marsh ${ }^{1,3}$, Helen D. \\ Brasch $^{1}$, Swee T. Tan ${ }^{1,4, \#}$, Tinte Itinteang ${ }^{1, \#}$ \\ 'Gillies McIndoe Research Institute, Wellington 6242, New Zealand. \\ ¿Upper Gastrointestinal, Hepatobiliary \& Pancreatic Section, Department of General Surgery, Wellington Regional Hospital, \\ Wellington 6242, New Zealand. \\ ${ }^{3}$ University of Auckland, Auckland 1010, New Zealand. \\ ${ }^{4}$ Wellington Regional Plastic, Maxillofacial \& Burns Unit, Hutt Hospital, Wellington 6242, New Zealand. \\ \#Authors contributed equally.
}

Correspondence to: Dr. Swee T. Tan, Gillies McIndoe Research Institute, PO Box 7184, Newtown, Wellington 6242, New Zealand. E-mail: swee.tan@gmri.org.nz

How to cite this article: Narayanan A, Wickremesekera SK, van Schaijik B, Marsh RW, Brasch HD, Tan ST, Itinteang T. Cancer stem cells in liver metastasis from colon adenocarcinoma express components of the renin-angiotensin system. J Cancer Metastasis Treat 2019;5:36. http://dx.doi.org/10.20517/2394-4722.2018.77

Received: 20 Nov 2018 First Decision: 19 Jan 2019 Revised: 9 Feb 2019 Accepted: 28 Feb 2019 Published: 24 Apr 2019

Science Editor: Guo-Feng Xie Copy Editor: Cai-Hong Wang Production Editor: Huan-Liang Wu

\begin{abstract}
Aim: We have recently identified a cancer stem cell (CSC) subpopulations within the tumor nests (TNs) and another within the peritumoral stroma (PTS) in liver metastasis from colon adenocarcinoma (LMCA). This study investigated the expression of components of the renin-angiotensin (RAS): pro-renin receptor (PRR), angiotensin converting enzyme (ACE), and angiotensin II receptor 1 (ATIIR1) and angiotensin II receptor 2 (ATIIR2) in LMCA.

Methods: 3,3-Diaminobenzidine (DAB) immunohistochemical (IHC) staining was performed on 16 LMCA samples for PRR, ACE, ATIIR1 and ATIIR2. Immunofluorescence (IF) IHC staining was performed to investigate co-expression of these components of the RAS with SOX2 or OCT4. NanoString analysis $(n=6)$ and Western blotting $(\mathrm{WB}, n=3)$ were performed on snap-frozen LMCA samples to confirm mRNA and protein expression, respectively.

Results: DAB IHC staining showed the expression of PRR, ACE, ATIIR1 and ATIIR2 within all LMCA samples. NanoString analysis and WB confirmed gene and protein expression of these components of the RAS. IF IHC staining demonstrated expression of PRR, ATIIR1 and ATIIR2 by the SOX2 ${ }^{+} \mathrm{CSC}$ s within the TNs and the $\mathrm{OCT} 4^{+} \mathrm{CSCs}$ within the PTS. ACE was expressed on the endothelium of the microvessels within the PTS.
\end{abstract}

\footnotetext{
(@) (-)

(C) The Author(s) 2019. Open Access This article is licensed under a Creative Commons Attribution 4.0 International License (https://creativecommons.org/licenses/by/4.0/), which permits unrestricted use, sharing, adaptation, distribution and reproduction in any medium or format, for any purpose, even commercially, as long as you give appropriate credit to the original author(s) and the source, provide a link to the Creative Commons license, and indicate if changes were made.
}

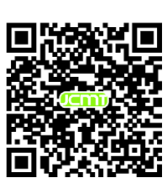


Conclusion: These finding suggests the CSCs within LMCA maybe a novel therapeutic target by manipulation of the RAS.

Keywords: Cancer stem cells, colorectal, colon, cancer, adenocarcinoma, liver, metastasis, renin-angiotensin system

\section{INTRODUCTION}

Colorectal cancer (CRC) accounts for 9.7\% of all cancers with approximately 1.5 million cases worldwide in $2015^{[1]}$, including 3158 cases in New Zealand ${ }^{[2]}$. It is the second most common cause of cancer death in the United Kingdom $^{[3]}$ and New Zealand ${ }^{[2]}$.

The pathogenesis of CRC relates to a complex interaction between genetic predisposition and lifestyle factors $^{[1]}$. There are hereditary and non-hereditary CRC sub-types, although the majority are sporadic and result from somatic mutation in response to environmental factors ${ }^{[1]}$. A key molecular pathway in the development of about $80 \%$ of CRC cases is the adenocarcinoma sequence, in which an accumulation of genetic mutations, particularly in the adenomatous polyposis coli pathway leads to carcinogenesis ${ }^{[4]}$. Diets high in animal fat and red meat, physical inactivity and excess body weight, heavy alcohol consumption and smoking are known modifiable risk factors for $\mathrm{CRC}^{[5]}$.

The liver is commonly the first metastatic site for CRC, and may be the only site of spread in up to $30 \%-40 \%$ of patients with advanced disease ${ }^{[6]} .20 \%-25 \%$ of CRC patients have detectable synchronous liver metastases at the time of diagnosis, and another $40 \%-50 \%$ of patients will subsequently develop metachronous liver metastasis within three years of resection of the primary tumor ${ }^{[7]}$.

The current concept of CRC proposes that the primary mode of tumor spread is via the portal system, and hence resection of isolated liver metastasis from CRC adenocarcinoma may be curative ${ }^{[6]}$. However, the observation of circulating CRC cancer stem cells $(\mathrm{CSCs})^{[8]}$ infers that the assumption of the liver being the most suitable site for metastasis remains to be determined. The median survival of liver metastasis from CRC adenocarcinoma without treatment is less than 12 months from presentation, with improved prognosis for patients with isolated liver metastasis ${ }^{[9]}$. Approximately $20 \%-30 \%$ of patients with liver metastasis from CRC adenocarcinoma have disease confined to the liver and is therefore potentially operable ${ }^{[10]}$ with a fiveyear survival of $25 \%-44 \%^{[7,11]}$. There has been recent focus on pre-operative neo-adjuvant chemotherapy to improve disease-free and overall survival by downsizing metastatic liver disease to increase operability and treat systemic disease $e^{[12]}$.

The CSC concept of cancer proposes that a subpopulation of cells within a cancer that possesses the properties of embryonic stem cells (ESCs) are the driving force for carcinogenesis due to their innate ability to promote angiogenesis, local invasion, distant metastasis, and resistance to apoptosis ${ }^{[13]}$. Although the origin of CSCs remains to be elucidated, they are distinguishable from the cancer cell population by their expression of ESC markers, well researched in many cancer types including oral cavity squamous cell carcinoma (OCSCC) affecting different sub-sites ${ }^{[14-16]}$ and glioblastoma $(\mathrm{GB})^{[17]}$. Increased expression of ESC markers has been associated with increased tumor size, local invasion and metastasis ${ }^{[18]}$, worsening prognosis, treatment resistance ${ }^{[19]}$ and risk of local recurrence or metastasis ${ }^{[20]}$.

We have recently demonstrated the presence of three CSC subpopulations within liver metastasis from colon adenocarcinoma (LMCA): a $\mathrm{SOX}_{2}{ }^{+} / \mathrm{NANOG}^{+} / \mathrm{KLF}^{+} / \mathrm{c}-\mathrm{MYC}^{+} / \mathrm{OCT}$ subpopulation within the tumor nests (TNs), and a $\mathrm{SOX}_{2}{ }^{+} / \mathrm{NANOG}^{+} / \mathrm{KLF}^{+} / \mathrm{c}-\mathrm{MYC}^{+} / \mathrm{OCT}_{4}{ }^{-} \mathrm{CSC}$ subpopulation and a $\mathrm{SOX}^{+} / \mathrm{NANOG}^{+} / \mathrm{KLF}^{+} /$

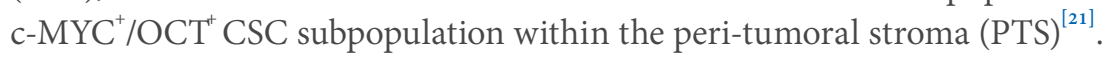

The renin-angiotensin system (RAS) is a hormonal system that is classically known for regulation of cardiovascular homeostasis and blood pressure ${ }^{[22]}$. A key component of the RAS is angiotensinogen which 
is physiologically released from the liver into the circulation and is converted to angiotensin I (ATI) by renin - the active form of the proenzyme, pro-renin. The receptor for both renin and pro-renin is pro-renin receptor $(\mathrm{PRR})^{[23]}$. ATI is then converted to angiotensin II (ATII) by angiotensin converting enzyme (ACE). The effects of ATII are mediated through its receptors, angiotensin II receptor 1 (ATIIR1) and angiotensin II receptor 2 (ATIIR2) ${ }^{[24]}$.

Recent studies show that the RAS promotes tumor growth at a local tissue level by modulating angiogenesis, tumor cell proliferation, immune responses and extracellular matrix formation ${ }^{[24]}$. Patients who are administered RAS modulators have a reduced incidence of CRC as well as lower rate of developing distant metastasis from $\mathrm{CRC}^{[25,26]}$.

CSCs in a number of cancer types, including $\operatorname{OCSCC}^{[16,27,28]}$ and $\mathrm{GB}^{[17]}$ have been shown to express components of the RAS. Hence CSCs may be a novel therapeutic target by modulation of the RAS. A recent meta-analysis reports an average $20 \%$ reduced risk of metastasis and improved survival from cancer including CRC, in patients who are administered RAS modulators ${ }^{[2]}$.

Currently there are few publications showing the presence of the components of the RAS in LMCA. This study aimed to investigate the expression of the components of the RAS: PRR, ACE, ATIIR1, and ATIIR2 within LMCA, in relation to the three CSC subpopulations we have recently identified ${ }^{[21]}$, using 3,3-diaminobenzidine (DAB) and immunofluorescence (IF) immunohistochemical (IHC) staining, Western blotting (WB), and NanoStrong mRNA expression analysis.

\section{METHODS}

\section{Tissue samples}

LMCA tissue samples from 16 male patients aged 50-80 (mean 65) years including those in our previous study ${ }^{[21]}$ were sourced from the Gillies McIndoe Research Institute Tissue Bank and used for this study which was approved by the Central Health and Disabilities Ethics Committee. Informed written consent was obtained from all participants.

\section{Histochemical and immunohistochemical staining}

Hematoxylin and eosin (H\&E) staining was carried out on $4 \mu \mathrm{m}$-thick formalin-fixed paraffin-embedded sections of 16 LMCA tissue samples to confirm the presence of the tumor on the slides by an anatomical pathologist (HDB). DAB IHC staining was then performed on these sections using the Leica Bond Rx auto-stainer (Leica, Nussloch, Germany) with primary antibodies for PRR (1:2000; cat\# AB40790, Abcam, Cambridge, MA, USA), ACE (1:100; cat\# MAB4399, AbD Serotec, Oxford, UK), ATIIR1 (1:300; cat\# AB9391, Abcam), and ATIIR2 (1:2000; cat\# NBP1-77368, Novus Biologicals, Littleton, CO, USA), OCT4 (1:30; cat\# MRQ-10, Cell Marque), SOX2 (1:200; cat\# PA1-094, Thermo Fisher Scientific, Rockford, IL, USA). Antibodies were diluted in Bond primary antibody diluent (Leica). All DAB IHC-stained slides were mounted in Surgipath Micromount (Leica, Nussloch, Germany). To confirm co-expression of two proteins, two representative samples of LMCA from the original cohort of 16 patients used for DAB IHC staining, underwent IF IHC staining. Vectafluor Excel anti-mouse 488 (ready-to-use; cat\# VEDK2488, Vector Laboratories, Burlingame, CA, USA) and Alexa Fluor anti-rabbit 594 (1:500; cat\# A21207, Life Technologies, Carlsbad, CA, USA) were used to detect the combinations. All IF IHC-stained slides were mounted in Vecta Shield Hardset mounting medium with 4, 6-diamino-2-phenylindone (Vector Laboratories). All antibodies were diluted in Bond primary diluent (Leica). All DAB and IF IHC staining was performed using the Leica Bond Rx auto-stainer (Leica), as previously described ${ }^{[16,28,30]}$.

\section{Image analysis}

The DAB IHC-stained slides were viewed and the images were captured using an Olympus BX53 light microscope fitted with an Olympus DP21 digital camera (Olympus, Tokyo, Japan). An Olympus FV1200 
confocal laser-scanning microscope (Olympus) was used for IF IH-stained slides and the images were processed with CellSens Dimension 1.11 software using the 2D deconvolution algorithm (Olympus).

\section{NanoString mRNA expression analysis}

Six snap-frozen LMCA tissue samples from the original cohort of 16 patients included for DAB IHC staining were used to isolate total RNA. RNA was extracted using the MagJET RNA Kit and KingFisher Duo (ThermoFisher Scientific) protocol and quantitated by the NanoDrop 2000 Spectrophotometer (Thermo Scientific) and Qubit (Thermo Scientific). mRNA was assayed by New Zealand Genomics Ltd (Dunedin, NZ), using the NanoString nCounter Gene Expression Assay (NanoString Technologies, Seattle, WA, USA). Probes for the genes were designed and synthesized by NanoString Technologies and are PRR (ATP6AP2, NM_005765.2), ACE (CD143, NM-000789.2), ATIIR1 (AGTR1, NM_000685.3), and ATIIR2 (AGTR2, NM_000686.3). Raw data were analyzed by nSolver software (NanoString Technologies) using standard settings and were normalized against the housekeeping gene glyceraldehyde 3-phosphate dehydrogenase $(\mathrm{GAPDH})$, as routinely performed in our laboratory ${ }^{[16,28]}$.

\section{Western blotting}

Total protein was extracted from three snap-frozen LMCA tissue samples from the original cohort of 16 patients used for DAB IHC staining, resolved by SDS-PAGE, and transferred to a PVDF membrane as previously described ${ }^{[31]}$, and probed using the following antibodies: PRR (1:500, cat\# ab40790, Abcam), ATIIR1 (1:500; cat\# sc-1173, Santa Cruz, CA, USA), ATIIR2 (1:1000; cat\# ab92445, Abcam), ACE (1:200; cat\# sc-12184, Santa Cruz), and $\beta$-actin (1:500; cat\# ab8229, Abcam). Secondary and tertiary antibodies were goat anti-rabbit HRP (1:2000; cat\# ab6721, Abcam) for PRR and ATIIR1, rabbit anti-goat Superclonal ${ }^{\mathrm{TM}}$ biotin conjugate (1:5000; cat\# A27013, Thermo Fisher) and goat anti-rabbit Superclonal ${ }^{\mathrm{TM}}$ biotin conjugate (1:5000; cat\# A27035, Thermo Fisher) followed by Pierce ${ }^{\mathrm{TM}}$ Streptavidin Poly-HRP (1:5000, cat\# 21140, Thermo Fisher) for ACE and ATIIR2, respectively, and chicken anti-goat Alexa Fluor 647 (1:2000; cat\# A21244, Life Technologies) for $\beta$-actin. Clarity Western ECL (cat\# 1705061, Bio-Rad) was used as the substrate for HRP bands and the Chemi Doc MP Imaging System (Bio-Rad) and ImageLab 5.0 software (Bio-Rad) were used for detection and analysis, as previously used ${ }^{[16,28]}$. Positive controls were snap-frozen human placenta tissue for PRR and ATIIR1, snap-frozen mouse lung tissue for ACE, and a recombinant ATIIR2 protein (cat\# Ho0000186-P01, Novus Biologicals) for ATIIR2. Matched mouse (1:500; cat\# ab18443, Abcam) and rabbit (1:500; cat\# ab171870, Abcam) isotype controls were used as appropriate negative controls.

\section{Statistical analysis}

Statistical analysis of the NanoString mRNA data was performed using $t$ test (SPSS v 24).

\section{RESULTS}

\section{Histology and DAB IHC staining}

H\&E staining confirmed the presence of LMCA on the slides for each of the 16 tissue samples. DAB IHC staining demonstrated the expression of PRR [Figure 1A, brown] which was localized to cells within the TNs, cells within the PTS and the endothelium of the microvessels within the PTS. ACE [Figure 1B, brown] was expressed on the luminal surface of the TNs and weakly on the endothelium of the microvessels within the PTS. Strong cytoplasmic expression of ATIIR1 [Figure 1C, brown] was present on the cells within the TNs, the cells within the PTS and the endothelium of the microvessels within the PTS. ATIIR2 [Figure 1D, brown] was also expressed by the cells within the TNs, and weakly on the endothelium of the microvessels within the PTS.

The expected expression patterns of positive controls for PRR [Supplementary Figure 1A, brown], ACE [Supplementary Figure 1B, brown], ATIIR1 [Supplementary Figure 1C, brown], and ATIIR2 [Supplementary Figure 1D, brown] were demonstrated in human placenta, kidney, liver and kidney, 

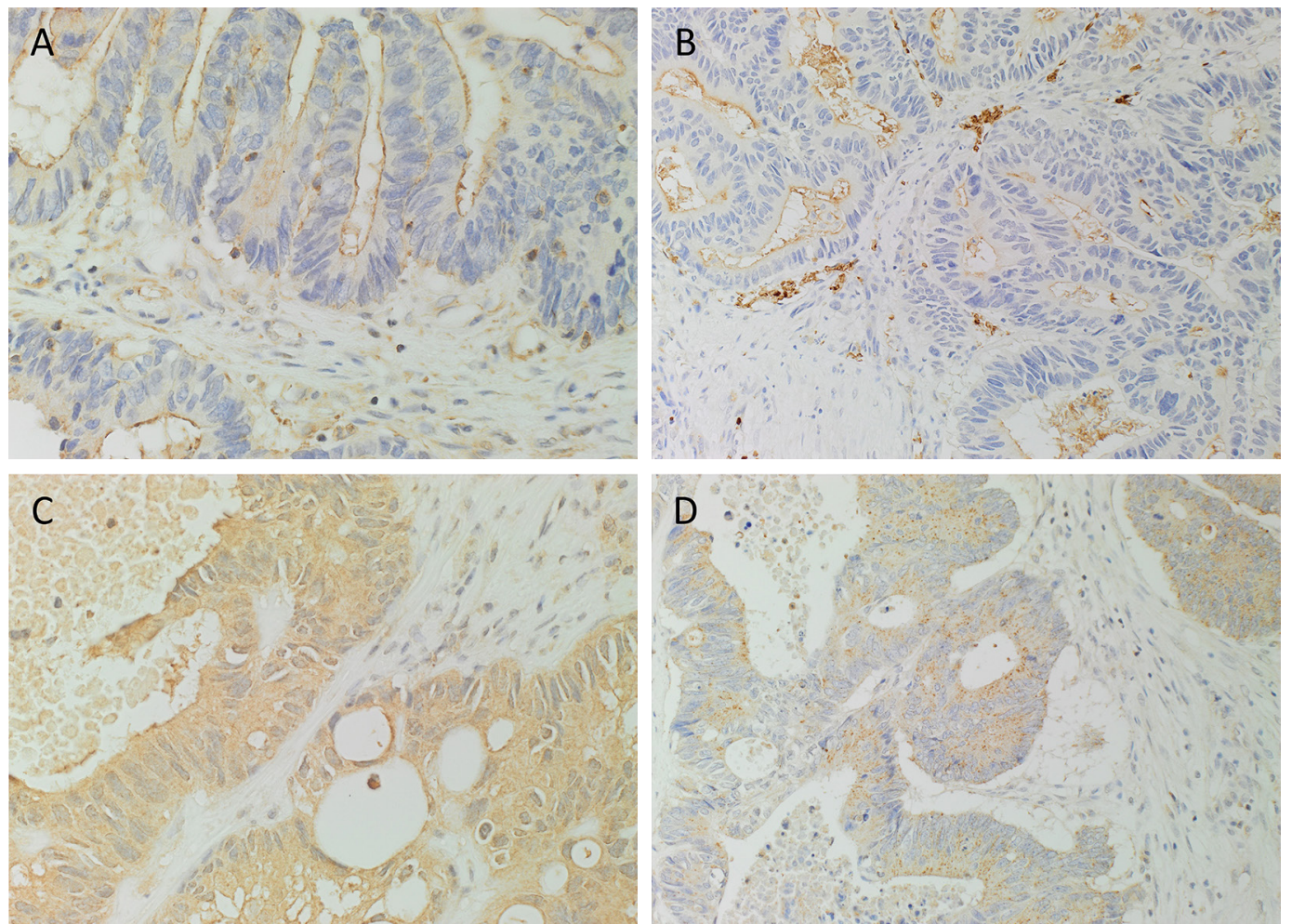

Figure 1. 3,3-Diaminobenzidine immunohistochemical-stained sections of liver metastases from colon adenocarcinoma demonstrating the expression of pro-renin receptor [(A), brown] localized to cells within the tumor nests (TNs), the cells within the peritumoral stroma (PTS) and the endothelium of the microvessels within the PTS. Angiotensin converting enzyme [(B), brown] was expressed on the luminal surface of the TNs and weakly on the endothelium of the microvessels within the PTS. Angiotensin II receptor 1 (ATIIR1) [(C), brown] demonstrated strong cytoplasmic expression on cells within the TNs, the endothelium of the microvessels and the cells within the PTS. ATIIR2 [(D), brown] was also expressed on the cells within the TNs and weakly on the endothelium of the microvessels within the PTS. Nuclei were counter-stained with hematoxylin [(A-D), blue]. Original magnification: 400x

respectively. The negative controls on LMCA tissue samples, stained using an IgG isotype [Supplementary Figure $1 \mathrm{E}]$, demonstrated the specificity of the primary antibodies used.

\section{IF IHC staining}

IF IHC staining was employed to investigate the expression of components of RAS by the CSC subpopulations within LMCA by co-staining with ESC markers OCT4 and SOX2. PRR [Figure 2A, red] was expressed by the cells within the TNs and the cells within the PTS, regardless of whether they were OCT4 ${ }^{+}$ [Figure 2A, green] or OCT4 [Figure 2A]. ACE [Figure 2B, green] was expressed on the luminal surface of the cells within the TNs as well as the endothelium of the microvessels within the PTS, which also expressed SOX2 [Figure 2B, red]. ATIIR1 [Figure 2C, green] was demonstrated in cells within the TNs, the endothelium of the microvessels within the PTS [Figure $2 \mathrm{C}$ ] and the cells within the PTS [Figure $2 \mathrm{C}$ ], which also expressed SOX2 [Figure 2C, red]. A similar expression pattern was seen for ATIIR2 [Figure 2D, red] in the cells within the TNs and the cells within of the PTS whether they expressed OCT4 [Figure 2D, green] or not [Figure 2D].

Split images of the stains presented in Figure 2 are shown in [Supplementary Figure 2]. Minimal staining was present on the negative control [Supplementary Figure 2I], confirming the specificity of the primary antibodies used.

\section{NanoString mRNA expression analysis}

NanoString mRNA expression analysis of PRR, ACE, ATIIR1, and ATIIR2 on six snap-frozen LMCA tissue samples of the original cohort of 16 patients used for DAB IHC staining, normalized against the 

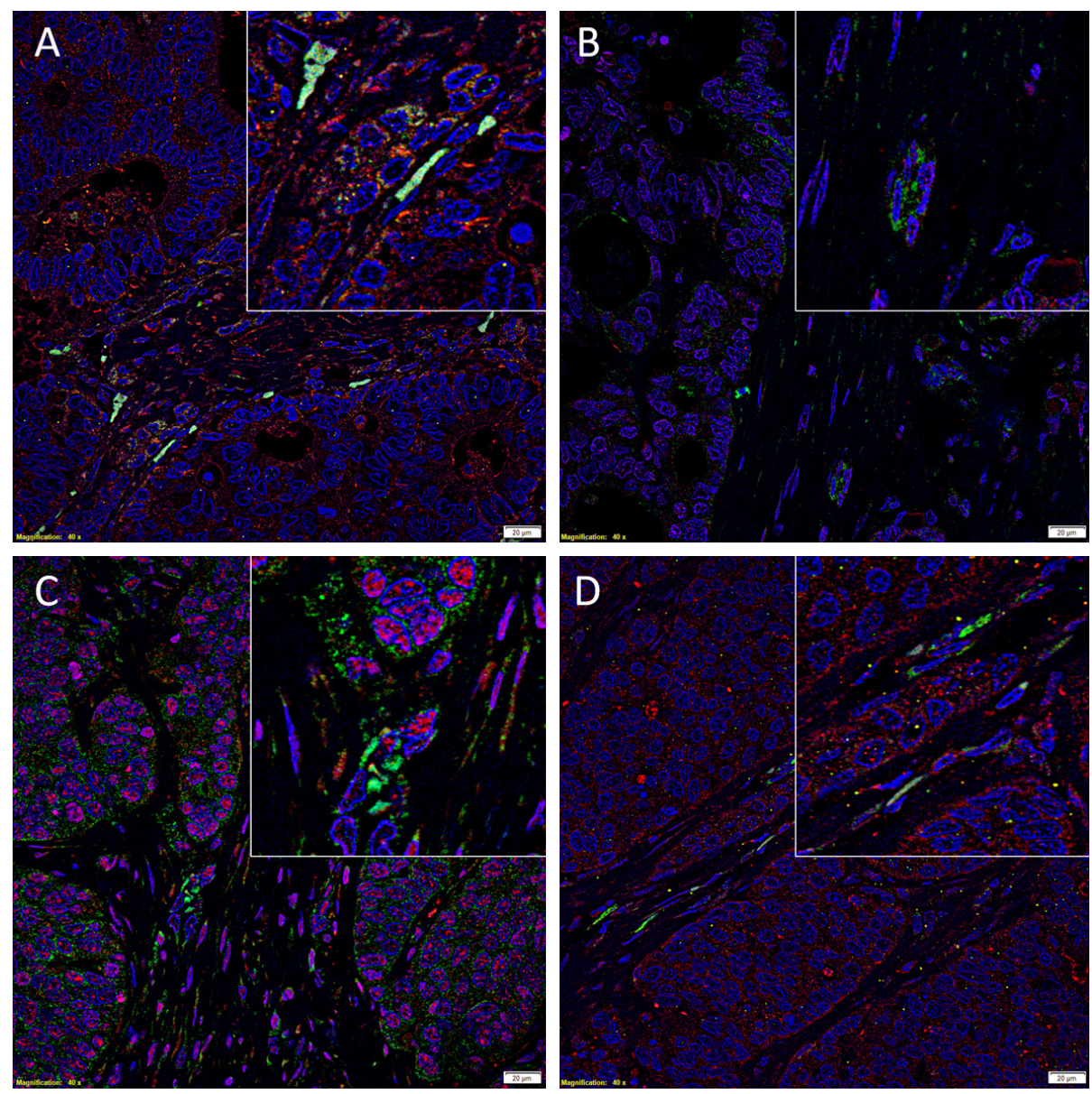

Figure 2. Representative immunofluorescence immunohistochemical-stained sections of liver metastasis from colon adenocarcinoma demonstrating the expression of pro-renin receptor [(A), red] by the cells within the tumor nests (TNs), the cells within the peritumoral stroma (PTS) regardless of whether they were $\mathrm{OCT4}^{+}[(\mathrm{A})$, green] or OCT4- (A). Angiotensin converting enzyme [(B), green] was expressed on the endothelium of the microvessels within the PTS, which also expressed SOX2 [(B), green]. Angiotensin II receptor 1 (ATIIR1) [(C), green] was demonstrated on cells within the TNs, the endothelium of the microvessels within the PTS (C) and the cells within the PTS (C), which also expressed SOX2 [(C), red]. A similar expression pattern was seen for ATIIR2 [(D), red] in the cells within the TNs and the cells within of the PTS whether they expressed OCT4 [(D), green] or not (D). All slides were counter-stained with 4',6'-diamino-2-phenylindole [(A-D), blue]. Scale bars: $20 \mu \mathrm{m}$; insert: $400 \times$ magnification

housekeeping gene GUSB, confirmed the presence of PRR, ACE and ATIIR1 mRNA in all six samples and ATIIR2 in one sample [Figure 3], with ATIIR2 mRNA expression at a significantly lower level in all six samples compared to the other genes $(P<0.05)$.

\section{Western blotting}

WB on the three snap-frozen LMCA tissue samples demonstrated the presence of bands at the expected molecular weight for PRR, ATIIR1, ATIIR2 and ACE [Figure 4]. PRR was detected in all three samples at $22 \mathrm{kDa}$ [Figure $4 \mathrm{~A}$ ], with the specificity of the antibody confirmed using a recombinant PRR protein (cat\# ab153053, Abcam). ATIIR1 was detected in all three samples at the expected molecular weight of 41 $\mathrm{kDa}$ [Figure $4 \mathrm{~B}$ ], and ATIIR2 was detected in two out of three samples at 50kDa [Figure $4 \mathrm{C}$ ], consistent with ATIIR2 variants from adrenal glands and from ATIIR2 proteins with high glycosylation ${ }^{[32]}$. ACE was detected at a low abundance in all three samples at the expected molecular weight of $194 \mathrm{kDa}$ [Figure $4 \mathrm{D}$ ]. This is supported by detection of ACE using the same antibody in different tissues ${ }^{[33]}$. $\beta$-actin confirmed approximate equivalent protein loading for all LMCA samples examined [Figure 4E]. The isotype controls are presented in Supplementary Figure 3. 


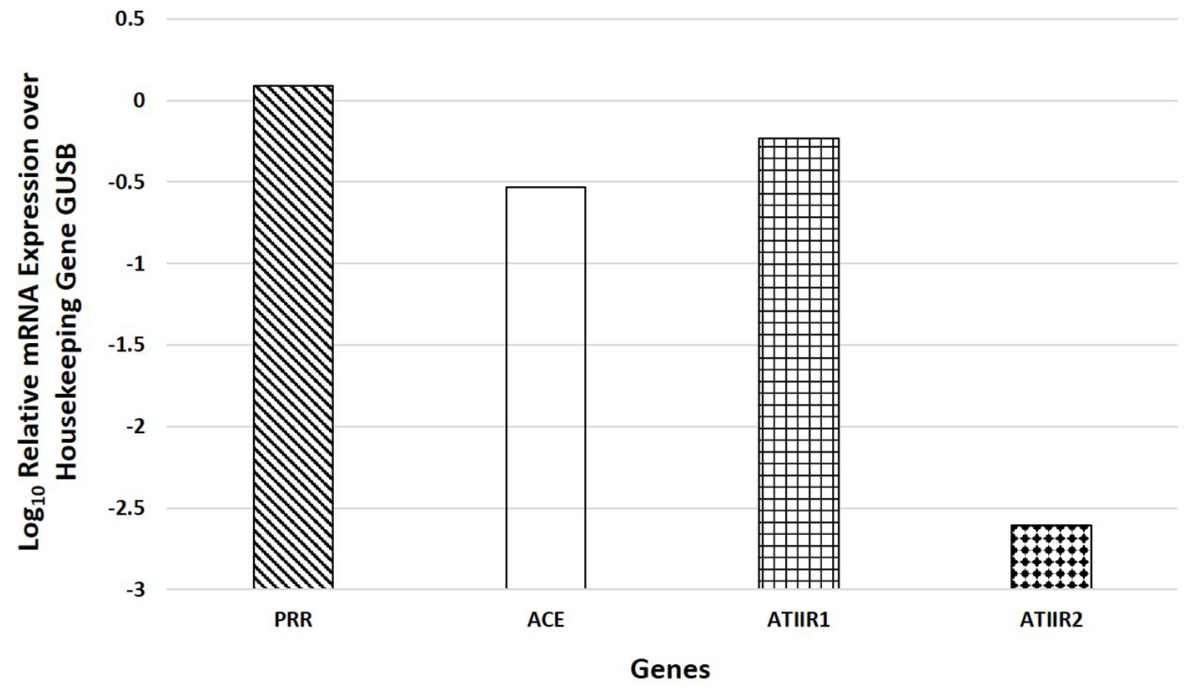

Figure 3. NanoString mRNA expression analysis of pro-renin receptor (PRR), angiotensin converting enzyme (ACE), angiotensin II receptor 1 (ATIIR1), and angiotensin II receptor 2 (ATIIR2) on six snap-frozen samples of liver metastasis from colon adenocarcinoma normalized against the housekeeping gene GUSB, confirmed the presence of PRR, ACE and ATIIR1 mRNA in all six samples and ATIIR2 in one sample, with ATIIR2 mRNA levels at a significantly lower level within all six samples compared to the other genes $(P<0.05)$

\section{DISCUSSION}

The classical RAS is an endocrine system that is crucial in regulating blood pressure and fluid homeostasis through its effects on the kidney, cardiovascular, and central nervous systems ${ }^{[24]}$. Physiologically angiotensinogen is cleaved by renin to form ATI. ACE then cleaves ATI to form ATII. ATII is the main effector of the RAS that mediates its effects by binding with its receptors ATIIR1 and ATIIR $2{ }^{[24]}$. Recent literature suggests a critical role for the RAS in cancer growth and metastasis ${ }^{[24]}$. There is increasing appreciation of the complex interplay between the RAS and other pathways including IGF, VEGF, and potential bypass loops consisting of cathepsins $B, D$ and $G^{[22,34]}$. These related pathways are implicated in a number of biological processes such as angiogenesis, cell migration, tumorigenesis and hematopoiesis which may create a conducive environment for carcinogenesis by promoting the proliferation of $\mathrm{CSCs}^{[22]}$.

More specifically to LMCA, a report by Heinzerling et al. ${ }^{[26]}$ shows the use of ACE inhibitors independently predicts less likelihood of patients with stage II CRC to develop distant metastasis. Using a murine model of CRC liver metastasis, Wen et al. ${ }^{[35]}$ show that administration of captopril, an ACE inhibitor, reduces proliferation of the CRC cells. These coupled with a recent review, highlighting the role of ACE inhibitors and ATIIR1 blockers in reducing of the incidence of CRC and the development of distant metastasis from $\mathrm{CRC}^{[25]}$. Taken together it is exciting to speculate that the expression of components of the RAS by the CSCs in LMCA is crucial in the maintenance and proliferation of these CSCs, although this remains the topic of future investigation.

We have recently demonstrated three subpopulations of CSCs within LMCA, including an OCT4 ${ }^{+}$ subpopulation, and an OCT4 subpopulation within the PTS and the TNs, suggesting the presence of a CSC hierarchy ${ }^{[21]}$. In this study we have demonstrated the expression components of the RAS: PRR, ACE and ATIIR1 and ATIIR2 by these CSC subpopulations within LMCA. It is interesting that ATIIR2 was detected by DAB IHC staining in all 16 samples, but only two out of the three samples by WB and in only one sample by NanoString mRNA analysis, possibly due to sampling bias.

The novel findings in this study mirror similar findings in OCSCC affecting the oral tongue ${ }^{[28]}$, buccal mucosa ${ }^{[27]}$ and lip ${ }^{[16]}$, and $\mathrm{GB}^{[17]}$. The expression of ACE on the endothelium of the microvessels within the 

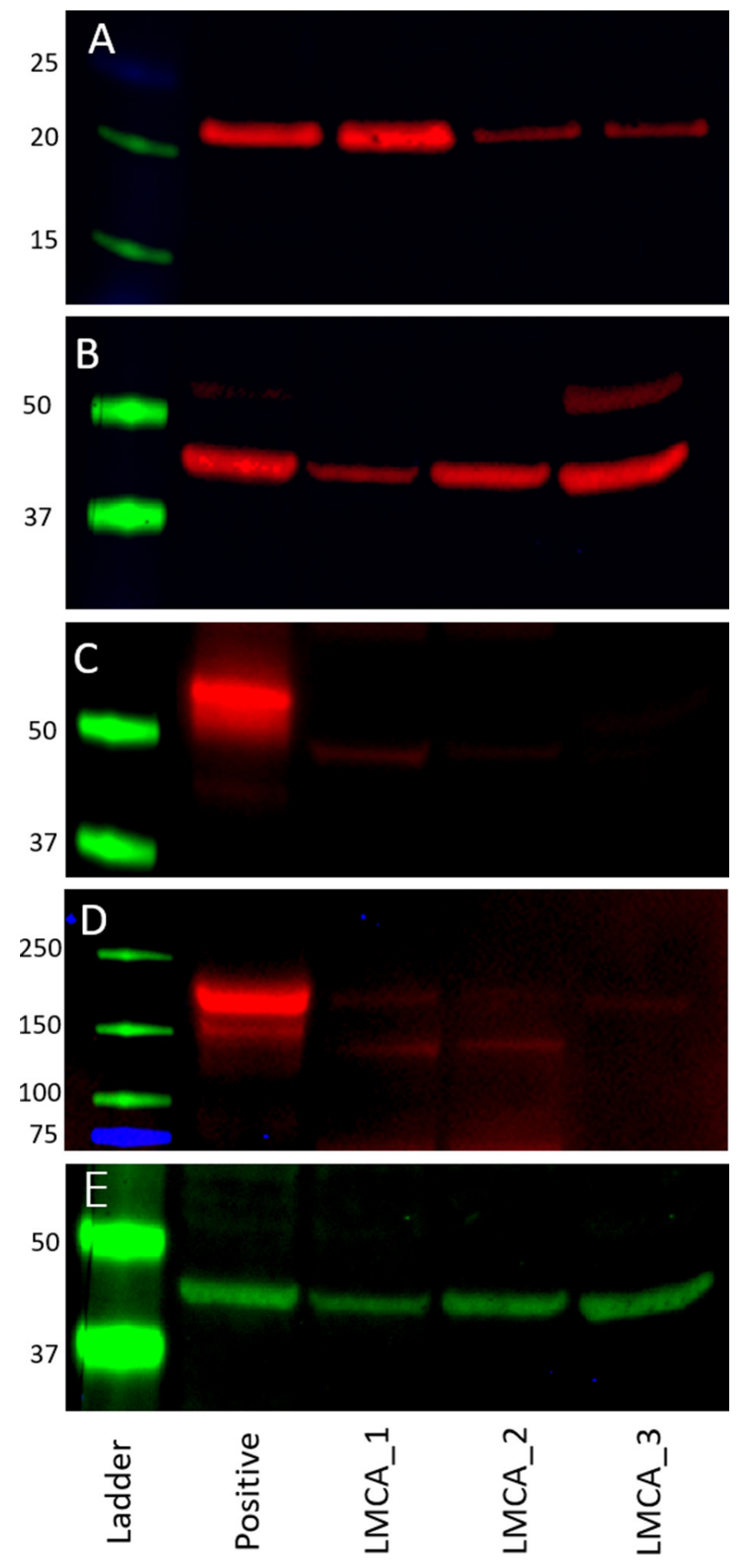

Figure 4. Western blot images of total protein extracted from snap-frozen samples of liver metastasis from colon adenocarcinoma (LMCA) from three patients demonstrating the presence of pro-renin receptor (PRR) in all three samples at $22 \mathrm{kDa}(\mathrm{A})$, with the specificity of the antibody confirmed using a recombinant PRR protein. Angiotensin II receptor 1 was detected in all three samples at the expected molecular weight of $41 \mathrm{kDa}(\mathrm{B})$; angiotensin II receptor 2 was detected in two out of three samples at $50 \mathrm{kDa}$ (C); angiotensin converting enzyme was detected at a low abundance in all three samples at the expected molecular weight of $194 \mathrm{kDa}(\mathrm{D})$; and $\beta$-actin confirmed approximate equivalent protein loading for all LMCA samples examined (E)

PTS suggests a role in regulating epithelial-to-mesenchymal transition by influencing angiogenesis ${ }^{[16,24]}$, although this requires further investigation. 
It is exciting to speculate that CSCs may be a novel therapeutic target by modulation of the RAS using existing medications such as aliskiren which targets renin, $\beta$-blockers which inhibit the production of prorenin and hence decrease renin levels, ACE inhibitors and ATII receptor blockers ${ }^{[36-38]}$. A recent metaanalysis on the use of ACE inhibitors and ATIIR1 blockers demonstrate an average $20 \%$ improved survival or reduction of recurrence in different types of cancers, including primary $\mathrm{CRC}^{[29]}$, suggesting a crucial role of these drugs in tumorigenesis, potentially through modulation on the RAS, although this requires further investigation.

Cathepsins B, D and G are proteases that constitute bypass loops for the $\mathrm{RAS}^{[22]}$ and have been implicated in tumorigenesis and metastasis in CRC due to their ability to degrade extra-cellular matrix. We have shown the expression of cathepsin B and cathepsin D by the CSC subpopulations within oral tongue squamous cell carcinoma $^{[27]}, \mathrm{GB}^{[39]}$ and more recently $\mathrm{LMCA}^{[34]}$, implying the presence of bypass loops for the RAS.

The novel finding of the expression of the components of RAS and cathepsin B and cathepsin D by the CSC sub-populations within LMCA, provides insights into possible more effective modulation of the RAS in targeting the CSCs. Further studies including a larger sample size and in vivo functional experiments are needed to explore the functional role of the RAS.

In conclusion, the novel finding of the expression of PRR, ATIIR1 and ATIIR2 on the CSCs within the TNs and CSCs within the PTS, and the expression of ACE in the endothelium of the microvessels in LMCA suggests the CSCs may be a novel therapeutic target by manipulation of the RAS.

\section{DECLARATIONS}

\section{Acknowledgments}

We thank Mrs Liz Jones and Mr Nick Bockett at the Gillies and McIndoe Research Institute, for performing the immunohistochemical staining and photographing the DAB IHC slides, respectively.

\section{Authors' contributions}

Formulated the study hypothesis: Tan ST, Itinteang T

Designed the study: Wickremesekera SK, Tan ST, Itinteang T

Interpreted the DAB and IF IHC data: Narayanan A, Brasch HD, Tan ST, Itinteang T

Interpreted the NanoString mRNA expression data: Narayanan A, Wickremesekera SK, Tan ST, Itinteang T

Performed the WB analysis and interpreted the results: van Schaijik B

Performed the statistical analysis: Marsh RW

Drafted the manuscript: Narayanan A, Tan ST, Itinteang T

\section{Availability of data and materials}

Data supporting the findings of this study can be obtained by contacting the corresponding author.

\section{Financial support and sponsorship}

None.

\section{Conflicts of interest}

The authors declared that there are no conflicts of interest.

\section{Ethical approval and consent to participate}

This study was carried out with the approval of the Central Health and Disability Ethics Committee (Ref. 15/CEN/106) with written informed consent from all subjects in accordance with The Declaration of Helsinki. 


\section{Consent for publication}

Not applicable.

\section{Copyright}

(c) The Author(s) 2019.

\section{REFERENCES}

1. Aran V, Victorino AP, Thuler LC, Ferreira CG. Colorectal cancer: epidemiology, disease mechanisms and interventions to reduce onset and mortality. Clin Colorectal Cancer 2016;15:195-203.

2. New Zealand Ministry of Health. Selected Cancers 2013, 2014 \& 2015 (Provisional). Wellington: Ministry of Health; 2016.

3. Garden OJ, Rees M, Poston GJ, Mirza D, Saunders M, et al. Guidelines for resection of colorectal cancer liver metastases. Gut 2006;55:iii1-8.

4. Fearon ER. Molecular genetics of colorectal cancer. Annu Rev Pathol 2011;6:479-507.

5. Haggar FA, Boushey RP. Colorectal cancer epidemiology: incidence, mortality, survival, and risk factors. Clin Colon and Rectal Surg 2009;22:191-7.

6. Weiss L, Grundmann E, Torhorst J, Hartveit F, Moberg I, et al. Haematogenous metastatic patterns in colonic carcinoma: an analysis of 1541 necropsies. J Pathol 1986;150:195-203.

7. Scheele J, Stang R, Altendorf-Hofmann A, Paul M. Resection of colorectal liver metastases. World J Surg 1995;19:59-71.

8. Grillet F, Bayet E, Villeronce O, Zappia L, Lagerqvist EL, et al. Circulating tumour cells from patients with colorectal cancer have cancer stem cell hallmarks in ex vivo culture. Gut 2017;66:1802-10.

9. Lahr CJ, Soong SJ, Cloud G, Smith JW, Urist MM, et al. A multifactorial analysis of prognostic factors in patients with liver metastases from colorectal carcinoma. J Clin Oncol 1983;1:720-6.

10. Stangl R, Altendorf-Hofmann A, Charnley RM, Scheele J. Factors influencing the natural history of colorectal liver metastases. Lancet 1994;343:1405-10.

11. Cady B, Stone MD. The role of surgical resection of liver metastases in colorectal carcinoma. Semin Oncol 1991;18:399-406.

12. Zalinski S, Mariette C, Farges O. Management of patients with synchronous liver metastases of colorectal cancer. Clinical practice guidelines. Guidelines of the French society of gastrointestinal surgery (SFCD) and of the association of hepatobiliary surgery and liver transplantation (ACHBT). Short version. J Visc Surg 2011;148:e171-82.

13. Shaheen, Mousa A, Hegazy NA, Nada OH, Radwan NA, et al. Immunohistochemical expression of stem cell markers CD133 and Oct4 in colorectal adenocarcinoma. Egyptian Journal of Pathology 2014;34:44-51.

14. Baillie R, Itinteang T, Yu HH, Brasch HD, Davis PF, et al. Cancer stem cells in moderately differentiated oral tongue squamous cell carcinoma. J of Clin Pathol 2016;69:742-4.

15. Yu HH, Featherston T, Tan ST, Chibnall AM, Brasch HD, et al. Characterization of cancer stem cells in moderately differentiated buccal mucosal squamous cell carcinoma. Front Surg 2016;3:46.

16. Ram RS, Brasch HD, Dunne JC, Davis PF, Tan ST, et al. Cancer stem cells in moderately differentiated lip squamous cell carcinoma express components of the renin-angiotensin system. Front Surg 2017;4:30.

17. Bradshaw AR, Wickremesekera AC, Brasch HD, Chibnall AM, Davis PF, et al. Glioblastoma multiforme cancer stem cells express components of the renin-angiotensin system. Front Surg 2016;3:51.

18. Noto Z, Yoshida T, Okabe M, Koike C, Fathy M, et al. CD44 and SSEA-4 positive cells in an oral cancer cell line HSC-4 possess cancer stem-like cell characteristics. Oral Oncology;49:787-95.

19. Elsir T, Edqvist PH, Carlson J, Ribom D, Bergqvist M, et al. A study of embryonic stem cell-related proteins in human astrocytomas: identification of nanog as a predictor of survival. Int J Cancer 2014;134:1123-31.

20. Chinn S, Darr O, Peters R, Prince M. The role of head and neck squamous cell carcinoma cancer stem cells in tumorigenesis, metastasis, and treatment failure. Front Endocrinol (Lausanne) 2012;3:90.

21. Humphries H, Wickremesekera SK, Marsh RW, Brasch HD, Mehrotra S, et al. Characterisation of cancer stem cells in metastatic colon adenocarcinoma to the liver. Front Surg 2018;4:76.

22. Munro MJ, Wickremesekera AC, Davis PF, Marsh R, Tan ST, et al. Renin-angiotensin system and cancer: a review. Integr Cancer Sci Ther 2017; doi: 10.15761/ICST:1000231.

23. Nguyen G. Renin, (pro) renin and receptor: an update. Clin Sci (Lond) 2011;120:169-78.

24. Ager EI, Neo J, Christophi C. The renin-angiotensin system and malignancy. Carcinogenesis 2008;29:1675-84.

25. Childers WK. Interactions of the renin-angiotensin system in colorectal cancer and metastasis. Int J Colorectal Dis 2015;30:749-52.

26. Heinzerling JH, Anthony T, Livingston EH, Huerta S. Predictors of distant metastasis and mortality in patients with stage II colorectal cancer. Am Surg 2007;73:230-8.

27. Featherston T, Yu HH, Dunne JC, Chibnall AM, Brasch HD, et al. Cancer stem cells in moderately differentiated buccal mucosal squamous cell carcinoma express components of the renin-angiotensin system. Front Surg 2016;3:52.

28. Itinteang T, Dunne JC, Chibnall AM, Brasch HD, Davis PF, et al. Cancer stem cells in moderately differentiated oral tongue squamous cell carcinoma express components of the renin-angiotensin system. J Clin Pathol 2016;69:942-5.

29. Sun H, Li T, Zhuang R, Cai W, Zheng Y. Do renin-angiotensin system inhibitors influence the recurrence, metastasis, and survival in cancer patients? Evidence from a meta-analysis including 55 studies. Medicine (Baltimore) 2017;96:e6394.

30. Tan EMS, Itinteang T, Chudakova DA, Dunne JC, Marsh R, et al. Characterisation of lymphocyte subpopulations in infantile 
haemangioma. J Clin Pathol 2015;68:812-8.

31. Tan K, Brasch HD, van Schaijik B, Armstrong JR, Marsh RW, et al. Expression and Localization of Cathepsins B, D, and G in Dupuytren's Disease. Plast Reconstr Surg Glob Open 9000 2018;6:e1686.

32. Harada K, Matsuoka H, Fujimoto N, Endo Y, Hasegawa Y, et al. Localization of type-2 angiotensin ii receptor in adrenal gland. J Histochem Cytochem 2010;58:585-93.

33. Hallersund P, Elfvin A, Helander HF, Fandriks L. The expression of renin-angiotensin system components in the human gastric mucosa. J Renin Angiotensin Aldosterone Syst 2011;12:54-64.

34. Mehrotra S, Wickremesekera SK, Schaijik BV, Brasch HD, Marsh RW, et al. Expression and localization of cathepsins B, D and G in cancer stem cells in liver metastasis from colon adenocarcinoma. Front Surg 2018;5:40.

35. Wen SW, Ager EI, Neo J, Christophi C. The renin angiotensin system regulates Kupffer cells in colorectal liver metastases. Cancer Biol Ther 2013;14:720-7.

36. Neal B, MacMahon S, Chapman N; Blood Pressure Lowering Treatment Trialists' Collaboration. Effects of ACE inhibitors, calcium antagonists, and other blood-pressure-lowering drugs: results of prospectively designed overviews of randomised trials. Lancet 2000;356:1955-64.

37. Oh BH, Mitchell J, Herron JR, Chung J, Khan M, et al. Aliskiren, an oral renin inhibitor, provides dose-dependent efficacy and sustained 24-hour blood pressure control in patients with hypertension. J Am Coll Cardiol 2007;49:1157-63.

38. Burnier M, Brunner HR. Angiotensin II receptor antagonists. Lancet 2000;355:637-45.

39. Koh SP, Wickremesekera AC, Brasch HD, Marsh R, Tan ST, et al. Expression of cathepsins B, D, and G in isocitrate dehydrogenasewildtype glioblastoma. Front Surg 2017;4:28. 